\title{
Editorial: Novel Smart Materials for Optical Fiber Sensor Development
}

\author{
Jin $\mathrm{Li}^{1,2 *}$, Duk-Yong Choi ${ }^{3,4}$ and Mateusz Smietana ${ }^{5}$ \\ ${ }^{1}$ College of Information Science and Engineering, Northeastern University, Shenyang, China, ${ }^{2}$ Hebei Key Laboratory \\ of Micro-Nano Precision Optical Sensing and Measurement Technology, Northeastern University at Qinhuangdao, \\ Qinhuangdao, China, ${ }^{3}$ Laser Physics Centre, Research School of Physics, Australian National University, Canberra, ACT, \\ Australia, ${ }^{4}$ College of Information Science and Technology, Jinan University, Guangzhou, China, ${ }^{5}$ Institute \\ of Microelectronics and Optoelectronics, Koszykowa, Warsaw, Poland
}

\section{INTRODUCTION}

In recent years, supercontinuum optical light sources and ultra-high sensitivity of various novel optical fibers or waveguides have been widely studied. Combined with the unique properties including low-loss transmission and anti-electromagnetic interference of optical fibers, a variety of photonic modulation and integrated all-optical sensing devices have been developed providing a possible technical path for integration of planar and fiber waveguides (Kosiel et al., 2018). Benefiting from the development of novel smart materials, nanoprocessing technologies, and optical spectra analysis techniques, many intelligent and high-performance optical waveguide devices or fiber sensors have been developed, in which, smart polymers, metal, metal oxide, and semiconductor materials have been used either to fabricate the optical fiber sensors or as the sensitive materials to effectively improve the sensitivity and selection performance (Yuan et al., 2019). This improvement is achieved through modifying different fiber structures such as microfiber, nanofiber, micro/nanostructures on fiber tip, multimode interference fiber structures, and in-line optical fiber structures. Optical fiber sensors at the micro/nanoscale have been integrated with microfluidic devices and planar photonic structures to develop alloptical chips, leading to high-speed acquisition, transmission, and processing of sensing signals. Because they are encapsulated in flexible materials, optical fiber sensors will be a promising candidate for use in wearable or implantable devices. Combining the excellent properties of micro/nanofiber (ultra-high evanescent field) with novel nanomaterials (high specific surface area and catalytic activity) used in these sensors has led to the development of many excellent integrated optical sensors. In this Research Topic, the latest research works covering both the model simulation and experimental studies on the structural design, device preparation, and sensing performance optimization of optical fiber sensors based on novel smart materials have been reported.

The flexible design and precise control of optical micro/nanofiber and micro/nanostructure are important supports for the development of advanced photonics devices and novel sensors, also named as "Lab on fiber" (Zhou et al., 2019). Dr. Liao et al. reviewed and discussed the research progress in the field of optical fiber integrated micro/nanostructures induced by two-photon polymerization in the last 10 years in their paper titled Optical Fiber Integrated Functional Micro/Nanostructure Induced by Two-Photon Polymerization. The ultra-compact and miniature microoptics, optical waveguide devices, and optical microcavities have been fabricated on the end face of optical fiber by laser micromachining, focused ion-beam milling, and nanoimprint technology with high resolution of less than $100 \mathrm{~nm}$. The combination of "Two-Photon Polymerization" technology, new processing methods or materials, and new functional structures have been contributing to the development of new nanophotonics devices, such as Lab on fiber. 
A variety of optical fiber structures are designed and used for sensing, relying on the effective interaction of the information between the optical signal and the environment to be measured ( $\mathrm{Li}$ et al., 2021). On the one hand, this process can be completed by limiting the gas/liquid in the optical fluid channel, such as photonic crystal fiber; on the other hand, the optical signal needs to be leaked to the outside of the fiber and contact with the environment to be measured using different fiber structures composited by dislocation fusion, microbending, micro/nanofiber, multimode interference, end reflection, and so on. Dr. Teng's group has designed a compact refractive index (RI) probe using the common single mode optical fiber by the low-cost fabrication process in their paper High-Sensitivity Refractive Index Sensor Based on a Cascaded Core-Offset and Macrobending Single-Mode Fiber Interferometer. A compact Mach-Zehnder interferometer (MZI) based on the cascaded core-offset and macrobending fiber structure is proposed and experimentally demonstrated with the sensitivity of $699.95 \mathrm{~nm} / \mathrm{RIU}$ at $\mathrm{RI}=1.43$ (phase demodulation method), as well as the high sensitivity of $366.78 \mathrm{~dB} / \mathrm{RIU}$ in the RI range of 1.35-1.38 (intensity demodulation method).

The detection process of traditional biochemical sensors is complex, involving the sample collection and long-time laboratory detection, so it is difficult to achieve rapid detection in in situ. Advanced spectral analysis technology and selective material modification on fiber probe can greatly shorten the detection time and improve the accuracy (Li J. et al., 2020). In Optical Fiber Sensors for Metal Ions Detection Based on Novel Fluorescent Materials, Dr. Cai reviewed the latest optical fiber sensors to determine the metal ions in liquid based on novel fluorescent materials, such as the organic dyes: protein, bacterial, and oligodeoxyribonucleotide or the typical inorganic quantum dots (QDs): semiconductor and carbon dots. The proposed sensors provide a promising approach for screening trace metal ions in environmental water samples. However, more works are desired on the fiber structure design, sensing system reform, quantitative method reconstruct, and fluorescent material immobilization.

\section{REFERENCES}

Kosiel, K., Koba, M., Masiewicz, M., and Śmietana, M. (2018). Tailoring properties of lossy-mode resonance optical fiber sensors with atomic layer deposition technique. Opt. Laser Technol. 102, 213-221.

Li, J., Wang, H., Li, Z., Su, Z., and Zhu, Y. (2020). Preparation and application of metal nanoparticals elaborated fiber sensors. Sensors 20:5155. doi: 10.3390/ s20185155

Li, J., Yan, H., Dang, H., and Meng, F. (2021). Structure design and application of hollow core microstructured optical fiber gas sensor: a review. Opt. Laser Technol. 135:106658. doi: 10.1016/j.optlastec.2020.106658

Li, Z., Liu, W., Cheng, H., Choi, D. Y., Chen, S., and Tian, J. (2020). Spin-selective full-dimensional manipulation of optical waves with chiral mirror. Adv. Mater. 32:1907983. doi: 10.1002/adma.201907983

Yuan, Z., Han, E., Meng, F., and Zuo, K. (2019). Detection and identification of volatile organic compounds based on temperature-modulated $\mathrm{ZnO}$ sensors. IEEE Trans. Instrum. Meas. 69, 4533-4544. doi: 10.1109/TIM.2019.2948413
At present, the expensive supercontinuum laser source has high power and is usually composed of multiple broad-spectrum light sources. It is only used in space optical system and is difficult to integrate with optical waveguide or fiber system (Li Z. et al., 2020). Prof. Bin Zhang proposed an on-chip mid-infrared (MIR) supercontinuum generation (SCG) with the ultra-wide spectral range of 3-12 $\mu \mathrm{m}$ in their work On-chip broadband midinfrared supercontinuum generation based on Highly non-linear chalcogenide glass waveguides. The structure of the waveguide onchip is similar to an optical fiber, consisting a "fiber core" (Ge-As$\mathrm{Se}-\mathrm{Te}$ ) and a "fiber cladding" (Ge-Se). The numerical model was proposed and demonstrated with the dispersive wave generation by optimizing the non-linear coefficients and dispersion profile. The fabrication method has also been designed to accurately control the dispersion property.

\section{CONCLUSION}

This special issue contains four articles, but we believe that the research on the sensitization mechanism and structure of advanced optical fiber integrated devices and novel materials will attract more extensive research interests of the researchers from the interdisciplinary fields such as biochemistry, materials, mechanics, and electricity.

\section{AUTHOR CONTRIBUTIONS}

JL performed the manuscript writing. D-YC and MS were involved in the manuscript review and editing. All authors contributed to the article and approved the submitted version.

\section{FUNDING}

This work is financially supported by the National Key R\&D Program of China (2019YFB2006001), Fundamental Research Funds for Central Universities (N2004007), and Hebei Natural Science Foundation (F2020501040).

Zhou, P., Liao, C., Li, Z., Liu, S., and Wang, Y. (2019). In-fiber cascaded FPI fabricated by chemical-assisted femtosecond laser micromachining for microfluidic sensing applications. J. Lightwave Technol. 37, 3214-3221. doi: 10.1364/ JLT.37.003214

Conflict of Interest: The authors declare that the research was conducted in the absence of any commercial or financial relationships that could be construed as a potential conflict of interest.

Copyright (c) $2021 \mathrm{Li}$, Choi and Smietana. This is an open-access article distributed under the terms of the Creative Commons Attribution License (CC BY). The use, distribution or reproduction in other forums is permitted, provided the original author(s) and the copyright owner(s) are credited and that the original publication in this journal is cited, in accordance with accepted academic practice. No use, distribution or reproduction is permitted which does not comply with these terms. 